\section{AI-ASSISTED WHOLE-BODY ASSESSMENT OF IMMUNOTHERAPY RESPONSE USING [18F]F-ARAG, A PET AGENT FOR ACTIVATED T CELLS}

${ }^{1}$ Jelena Levi", ${ }^{2}$ Timothy Perk, 'Lyna Huynh, ' ${ }^{1}$ Juliet Packiasamy, ${ }^{3}$ Serena Cheng, ${ }^{3} J o h n$ Sunwoo, ${ }^{3} \mathrm{~A}$ Dimitrios Colevas. ${ }^{1} \mathrm{Cell}$ Sight Technologies, San Francisco, CA, USA; ${ }^{2}$ AlQ Solution, Inc., Madison, WI, USA; ${ }^{3}$ Stanford University, Palo Alto, CA, USA

Background Patterns of response to immunotherapy differ from traditional cytotoxic drugs, making clinical decisions concerning response evaluation more challenging. Radiological response criteria, such as iRECIST, updated to address response patterns unique to immunotherapeutics, focus only on changes in the tumor burden. ${ }^{1}$ An $18 \mathrm{~F}$ labeled nucleoside analog, [18F]F-AraG was developed as a PET agent for imaging activated $\mathrm{T}$ cells, ${ }^{2-5}$ critical components of immune response and the common target of immunotherapies. Whole body assessment of [18F]F-AraG uptake that indicates presence of activated $\mathrm{T}$ cells might allow for analysis of complex immunologic processes and provide early indication of treatment response as well as off-target side effects. Here, we employ AIQ Solutions' TRAQinform IQ software to analyze [18F]FAraG scans and assess activation of $T$ cells in head and neck squamous cell carcinoma (HNSCC) patients undergoing antiPD-1 therapy.

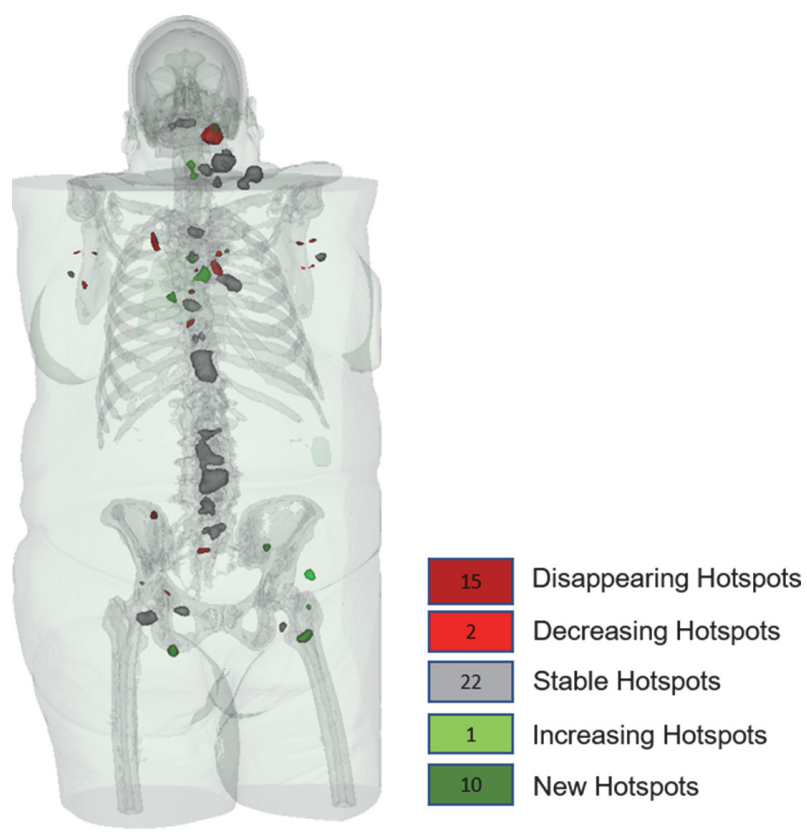

Abstract 45 Figure 1 TRAQinform IQ assessment of the SUVmean [18F]F-AraG signal change in a HNSCC patient two weeks after a single dose of anti-PD-1 antibody. Quantification of the differences in the [18F]F-AraG uptake in the baseline and on-treatment scan revealed 17 hotspots with disappearing or decreasing signal, 22 hotpots with the stable signal and 11 hotspots with increasing or new signal post therapy.

Methods Four treatment-naïve HNSCC patients were imaged using [18F]F-AraG before and 2-3 weeks after a single dose of anti-PD-1 antibody. [18F]F-AraG PET scans of six healthy subjects were used to define areas of increased [18F]F-AraG uptake in patients. The regions of uptake in the baseline and on-treatment scans were registered and matched using articulated registration. ${ }^{6} 7$ Standardized uptake values, SUVmax, SUVmean and SUVtotal were extracted from all areas of tracer uptake in both scans and changes in signal calculated to assess therapy effects. The change in signal was analyzed in the context of the patient's clinical status and changes in $T$ cell infiltration in the primary lesion when biopsy specimens were available.

Results TRAQinform IQ whole body evaluation of [18F]FAraG PET revealed heterogeneity in the signal range and extent of signal change both between different patients and between different areas of tracer uptake within the same patient (figure 1). The post-therapy whole-body [18F]F-AraG signal change trended with patients' outcome. The patients with areas where the signal disappeared or decreased post therapy, indicative of the lack of $\mathrm{T}$ cell activation, had shorter overall survival than the patients with areas with stable and increasing signal. The change in infiltration of activated $\mathrm{T}$ cells in the primary lesion tissue did not correspond to the patient survival, reflecting limitations of serial biopsy in evaluating therapy response.

Conclusions TRAQinform IQ analysis and quantification of [18F]F-AraG PET provides patient-level assessment of tracer uptake and may allow for better understanding of heterogeneity of $\mathrm{T}$ cell activation and potentially offer a more comprehensive evaluation of response to immunotherapy than the standard, tumor-centric, radiologic methods.

\section{REFERENCES}

1. Nishino $\mathrm{M}$, et al. Monitoring immune-checkpoint blockade: response evaluation and biomarker development. Nat Rev Clin Oncol, 2017.

2. Namavari $M$, et al. Synthesis of $2^{\prime}$-deoxy-2'-[18F]fluoro-9-beta-D-arabinofuranosylguanine: a novel agent for imaging T-cell activation with PET. Mol Imaging Biol 2011; 13(5):812-8.

3. Ronald JA, et al. A PET imaging strategy to visualize activated $T$ cells in acute graft-versus-host disease elicited by allogenic hematopoietic cell transplant. Cancer Res 2017; 77(11):2893-2902.

4. Levi J, et al. Imaging of activated $T$ cells as an early predictor of immune response to anti-PD-1 Therapy. Cancer Research 2019; 79(13):3455-3465.

5. Levi J, et al. (18)F-AraG PET for CD8 profiling of tumors and assessment of immunomodulation by chemotherapy. J Nucl Med 2021; 62(6):802-807.

6. Yip S, T Perk, and R Jeraj, Development and evaluation of an articulated registration algorithm for human skeleton registration. Phys Med Biol 2014; 59(6):148599.

7. Yip S. and R Jeraj. Use of articulated registration for response assessment of individual metastatic bone lesions. Phys Med Biol 2014; 59(6): 1501-14.

Ethics Approval The study was approved by Stanford University Ethics Board, approval number 40425.

http://dx.doi.org/10.1136/jitc-2021-SITC2021.045 